\title{
INVESTIGACION
}

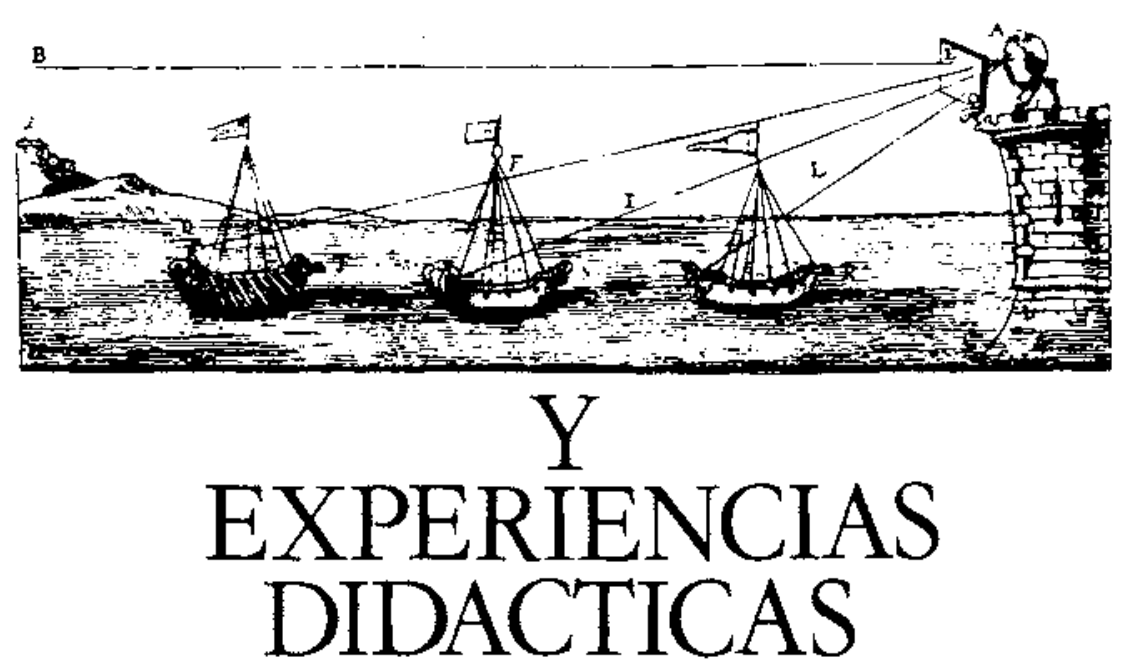

\section{IDEAS INTUITIVAS Y APRENDIZAJE DE LAS CIENCIAS}

SOLIS VILLA, R.

Departamento de Formación del Profesorado

ICE Universidad de Sevilla

\section{SUMMARY}

Current research in science education has shown that before any formal science teaching takes place, pupils have already acquired considerable knowledge about natural phenomena. This knowledge differs significantly from the accepted scientific view and interfere with the learning of the subject matter.

In this paper, the origin, characteristics and modification of pupils' intuitive ideas will be explored following the PSHG model (PSHG stands for Posner, Strike, Hewson and Gertzog); in terms of this model the process of learning science is a process of conceptual change from one set of concepts to another set, which is incompatible with the previous one.

\section{INTRODUCCION}

En los últimos ar̉os la investigación sobre aprendizaje y enseñanza de las ciencias se ha interesado en el estudio de las ideas intuitivas de los alumnes acerca de los fenómenos naturales y sus causas.

Bajo denominaciones distintas -errores conceptuales-(Linke y Venz, 1979); esquemas alternativos (Easley y Driver, 1978); conceptos alternativos
(Gilbert, 1983) - que reflejan posiciones epistemológicas diferentes, hay un hecho común: los alumnos no llegan como una tabula rasa; cada uno trae una estructura cognitiva, elaborada a partir de la experiencia diaria, que le sirve para explicar y predecir lo que ocurre a su alrededor.

Los resultados de investigaciones recientes muestran 
que las ideas intuitivas de los alumnos difieren significativamente del contenido formal de las asignaturas, interfiriendo en su aprendizaje. (Véase selección bibliográfica de Carrascosa, 1983).

Como consecuencia se dedica en la actualidad una atención creciente al origen, persistencia y modificación de los esquemas alternativos, junto a las implicaciones que se derivan para el diseño de los programas de las asignaturas, estrategias de enseñanza-aprendizaje y formación del profesorado de ciencias.

Este artículo va a tratar sobre el origen y caracteristicas de las ideas intuitivas, de su resistencia al cambio y de la interferencia que causan en el aprendizaje; se utilizará como marco de referencia el modelo PSHG, propuesto por Posner, Strike, Hewson y Gertzog, cuyas iniciales dan nombre al modelo, uno de los que gozan en la actualidad de mayor aceptación por su poder explicativo acerca del proceso de aprendizaje de las ciencias.

El análisis detallado de las implicaciones para el diseño de los programas y para la formación del profesorado de ciencias exigiría un trabajo que rebasaria las limitaciones de espacio de un articulo de revista.

\section{CARACTERISTICAS E IMPORTANCIA DE LAS IDEAS INTUITIVAS}

Antes de iniciar el estudio formal de las ciencias, los alumnos tienen ideas intuitivas acerca del mundo; este hecho se acepta desde diferentes posiciones, Ausubel (1968), Kelly (1971), Piaget (1965). Asi desde la perspectiva de la psicologia constructivista, estas ideas son, según Driver (1981), elaboraciones activas de la realidad a partir de:

- Un proceso combinado de inducción, intuición e imaginación del alumno.

- El uso de términos científicos en el lenguaje común.

- La influencia del entorno.

Los resultados de las investigaciones coinciden en varios aspectos sobre las características e importancia de la ideas intuitivas, (Osborne y Wittrock, 1983):

$1^{\circ}$ No son en general congruentes con los conceptos, leyes y teorías que los alumnos tienen que aprender.

$2^{\circ}$ Constituyen un esquema conceptual coherente, con amplio poder explicativo.

$3^{\circ}$ Son muy resistentes al cambio; a veces no cambian en absoluto, incluso después de varios años de contacto formal con las asignaturas. Cuando se produce el cambio, como resultado del estudio, puede no coincidir con lo previsto por el profesor.

$4^{\circ}$ Interfieren en el aprendizaje de las ciencias, siendo responsables en parte de la dificultad que encuentran los alumnos en estas asignaturas y de su bajo rendimiento comparado con otras áreas.

En realidad las dos últimas conclusiones son consecuen. cia de las dos primeras. Prescindir de un marco con- ceptual que ha servido para interpretar y dar sentido a la experiencia diaria, sustituyéndolo por otro con el que está en conflicto, es tarea difficil, cuando la realidad inmediata aparentemente concuerda con el esquema intuitivo.

A modo ilustrativo se enumeran algunas de las ideas intuitivas detectadas en investigaciones transculturales (Viennot, 1979), (Tasker, 1981), (Watts, 1983):

i un bloque permanece en reposo en un plano inclinado porque hace fuerza para no caer.

ii la fuerza es algo que hay dentro del objeto que se mueve.

iii un objeto necesita una fuerza continua para manzenerlo en movimiento.

iv la corriente eléctrica que fluye de la batería se va gastando.

$v$ las burbujas de agua hirviendo son burbujas de aire.

vi lá llama del mechero consume el gas.

¿Por qué difieren estas ideas de las aceptadas por la ciencia actual? La causa estriba en la forma en que los alumnos interpretan la realidad y en los factores que intervienen, para dar origen a sus ideas intuitivas.

\subsection{Ideas intuitivas y conocimiento científico}

Los niños y adolescentes observan los fenómenos naturales, buscan relaciones causales y elaboran modelos explicativos y predictivos de la realidad, coherentes y estables; éstos son diferentes de los modelos y teorias cientificas por varias razones, (Gilbert, 1982), Osborne, Bell y Gilbert, 1983):

a) Los conceptos intuitivos se construyen paulatinamente desde la infancia, interpretándose la realidad de acuerdo con las formas de pensamiento propias del niño. Animismo, realismo, artiftcialismo, son formas de pensar infantiles, (Piaget, 1965 y 1973a). El animismo, tendencia a atribuir vida a los objetos inanimados, origina ideas como (i): un bloque permanece en reposo en un plano inclinado porque hace fuerza para no caer.

Del mismo modo, la tendencia a dotar de realidad fisica las acciones, estados y pensamientos (realismo), hace que surjan ideas como (ii): la fuerza es algo que hay dentro del objeto que se mueve; o bien explica que muchos alumnos consideren el equilibrio como una entidad real, contenida en los objetos, no como una descripción de su estado.

b) Los alumnos tienden más a las explicaciones aisladas de hechos especificos que a buscar leyes generales válidas para diferentes fenómenos; incluso no les preocupa que haya inconsistencia entre sus explicaciones; Watts (1983) describe hasta 8 conceptos alternativos de fuerza utilizados en situaciones diversas. Del mismo modo en una investigación en curso se han detectado en los alumnos de COU explicaciones ad hoc para distintos movimientos. 
c) Los alumnos tienen dificultad para el razonamiento abstracto. Tienden a considerar el aspecto concreto de la situación y ello les lleva a conclusiones intuitivas, basadas en la inducción a partir de la observación de la realidad inmediata.

La explicación intuitiva más evidente es, a menudo, equivocada, (Einstein e Infeld, 1963). La realidad inmediata a menudo enmascara parte del fenómeno natural, que sólo se desvela en su totalidad por su proceso de abstracción hipotético-deductivo, que facilita la interpretación correcta del fenómeno. El ejemplo (iii) es ilustrativo: La observación diaria induce a pensar que un cuerpo se detiene cuando la fuerza que lo empuja deja de actuar. Este esquema alternativo, que relaciona la fuerza con la velocidad (si $F=0$, entonces $v=0$ ), es el resultado de un proceso de razonamiento basado en la inducción desde la realidad inmediata. Hay que abstraerse de ésta, eliminando las fuerzas de rozamiento, para llegar a la formulación de la $1^{\mathrm{a}}$ y $2^{\mathrm{a}}$ ley del movimiento de Newton ( $\mathrm{Fi}=0$, entonces $v=c t e$.) Fue precisamente el uso de la capacidad de abstracción, permitiendo el experimento ideal, lo que posibilitó el tránsito de la Física aristotélica a la Física de Galileo y Newton y ello llevó siglos. No es de extrañar que los alumnos adquieran y retengan estas ideas intuitivas pre-galileanas acerca del movimiento, que dificultan el aprendizaje de la Dinámica.

d) Muchos términos científicos están incorporados al lenguaje diario, con significados diferentes, ambiguos e incluso intercambiables. Trabajo, energía, fuerza, equilibrio, ácido, sales, corriente eléctrica... son de uso común y frecuentemente polivalente. Ello favorece la adquisición de los esquemas alternativos en el alumno y dificulta el aprendizaje.

Diferentes autores, Clement (1978), Viennot (1979), Watts (1983) han detectado el uso indistinto de las palabras fuerza y energia, lo que también puede interpretarse como una manifestación de la persistencia del concepto pre-galileano de impetus en los alumnos.

\subsection{Efecto de la enseñanza sobre las ideas intuitivas. Resistencia al cambio. Modelos explicativos del apren- dizaje de las ciencias.}

En principio podria suponerse que al estudiar las distintas asignaturas las ideas intuitivas desaparecen; sin embargo éstas persisten en muchos alumnos de diferentes edades, incluso universitarios, dificuliando el aprendizaje, (Driver, 1981 y Gilbert, 1983).

Esta resistencia al cambio de los esquemas alternativos está cuestionando el enfoque actual de la enseñanza de las ciencias. Entre los especialistas en este campo se trabaja en la elaboración de modelos que expliquen el aprendizaje de las ciencias partiendo de la estructura cognitiva previa del alumno.

\subsubsection{El modelo PSHG}

Posner y col. (1982) proponen un modelo (PSHG) que concibe el aprendizaje como un cambio paradigmático revolucionario en el sentido de Kuhn (1973); aprender significativamente ei contenido formal de las ciencias equivale a sustituir un paradigma (ideas intuitivas), familiar, inteligible y útil, por otro nuevo; éste será difícil de aceptar a menos que sea iguaimente inteligible que el antiguo y sea capaz de explicar hechos y resolver problemas que el anterior no resuelve.

El modelo PSHG parte del hecho de que los alumnos tienen un marco conceptual al que se refieren cada vez que se les presenta información nueva (ideas, experiencias, problemas). Estos conocimientos previos, cuya génesis ya se ha comentado, son inteligibles y útiles; inteligibles porque forman una estructura coherente, internamente consistente, representada en la memoria semántica del alumno y elaborada de acuerdo con su experiencia pasada; útiles porque sirven para interpretar la realidad presente y predecir lo que ocurre a su alrededor.

El aspecto central del modelo es analizar el proceso de enseñanza-aprendizaje como una interacción entre las ideas previas del alumno y la información nueva. $\mathrm{Pa}$ ra Posner y colaboradores, el aprendizaje es una actividad racional muy parecida al proceso de investigación científica, tratándose en los dos casos de un pro. ceso de cambio conceptual.

\section{Asimilación y acomodación}

Posner y col. (1982) usan estos dos términos para describir las distintas posibilidades de cambio conceptual, aunque los autores aclaran que si bien han tomado estos términos de Piaget, no significa que se adhieren a sus teorias.

a) Asimilación. Tiene lugar cuando los alumnos utilizan sus ideas previas para tratar la información nueva. Aprender un concepto, resoiver un problema, interpretar una experiencia, son diversas formas de tratar información nueva; para ello el alumno acude a su esquema conceptual previo; si el concepto le parece inteligible respecto a los que constituyen su esquema previo, si éste le permite resolver el problema y/o interpretar el experimento, el conocimien. to que resulta queda incorporado (asimilado) a su esquema conceptual previo.

b) Acomodación. Tiene lugar cuando el esquema conceptual previo es inadecuado para tratar la información nueva. Un concepto nuevo que no parece inteligible respecto al esquema conceptual previo, un problema que no se puede resolver o un experimento que no se puede interpretar, constituyen un reto para el esquema conceptual previo, lo que Kuhn denomina una anomalía. La aparición de anomalias crea en el alumno urił insatisfacción con sus ideas previas y como necesita un marco conceptual de referencia busca una solución, que puede con- 
sistir en una reestructuración o una sustitución de los conceptos existentes (acomodación), producièndose el cambio conceptual.

- La reestructuración consiste en una reorganización de los conceptos previos; utilizando una analogia se diría que se conservan los elementos que forman una red pero cambian las relaciones entre ellos. - La sustitución consiste en cambiar todas o parte de las ideas previas por otras nuevas. Posner opina que esta forma de acomodación constituye el verdadero cambio conceptual, aunque no es necesario que se sustituyan todos los conceptos previos; parte de éstos pueden mantenerse y servir de guia en el cambio de unas ideas por otras.

Los procesos de asimilación y acomodación pueden darse al mismo tiempo. Asi, parte del esquema conceptual previo se reorganiza, parte se sustituye por ideas nuevas y parte se modifica por simple incorporación (asimilación) de otras ideas nuevas. Ello equivale a considerar la estructura cognitiva de un alumno, no como un conjunto único y estático de conceptos, sino como una serie de subunidades internamente coherentes, relacionadas entre si, aunque a veces estos lazos de unión pueden ser muy débiles o incluso no existir; por efecto del aprendizaje la estructura en su conjunto va cambiando; no obstante los procesos de ásimilación y acomodación no son instantáneos, requieren tiempo, en especial la acomodación por las condiciones que este cambio supone.

\section{Condiciones para la acomodación}

La reorganización conceptual y sobre todo la sustitución de conceptos previos por otros nuevos, exige, según el modelo, que se den varias condiciones:

a) Debe existir insatisfacción con las ideas previas. $\mathrm{Pa}$ ra que se produzca insatisfacción con el esquema conceptual existente tiene que presentarse un número suficiente de anomalías que este esquema no pueda resolver.

b) Una idea nueva tiene que ser in teligible. Esta es una condición necesaria pero no suficiente. Para que una idea nueva sea inteligible ha de cumplir dos requisitos. Primero, hay que conocer y comprender los términos, símbolos y el modo de expresión (verbal, matenático, gráfico) utilizados; este es el primer paso para llegar a entender un concepto y en ocasiones no se cumple.

Segundo, se requiere darle un significado global a la información contenida en la idea nueva, esto es, estructurar de modo coherente la información; para ello el alumno trata de relacionar la idea nueva con ideas previas que le faciliten el proceso de estructuración, (Bruner, 1966). Una estrategia muy útil para relacionar información reciente (no viganizada) con los conocimientos existentes (información organizada) es el uso adecuado de analogías, metáforas y modelos, que permiten trasladar la in- formación nueva a un contexto familiar, favorecien. do el procesamiento de la información, y su consiguiente incorporación de forma organizada (una re. presentación mental coherente), no memorística, al esquema conceptual del alumno, (Belth, 1977).

c) Una idea nueva ha de ser verosimil. Entender una idea no es condición suficiente para aceptarla e incorporarla al esquema conceptual, además tiene que ser consisiente con las ideas previas, no estar en contradicción con ellas, ni con la experiencia diaria del alumno. Este requisito de ser creible es muy difícil de cumplir cuando la idea nueva aparece como contraintuitiva, siendo un obstáculo serio para el apren. dizaje. Así, puede resultar inteligible para el alumno la afirmación "al frenar un autobus bruscamente en realidad no hay ninguna fuerza que empuje hacia adelante al pasajero", pero de acuerdo con la experiencia diaria del alumno la idea le parecerá contraintuitiva; será necesario tiempo suficiente de reflexión sobre ejemplos y contraejemplos hasta que el alumno la encuentre verosímil.

d) Una idea nueva debe ser útil. Además de ser inteli. gible y verosimil, para aceptar una idea nueva, ésta debe servir para resolver las anomalias encontradas y ampliar el campo de conocimientos del alumno, sugiriéndole preguntas acerca de lo que observa, dandole respuestas satisfactorias y orientándolo hacia nuevos experimentos para verificar estas respues. tas; sólo así se producirá la aceptación de la idea nueva.

Las características de satisfactorio, inteligible, verosimil y útil, no son atributos discretos que el modelo asig. ne según el criterio del todo o nada. Un concepto in. tuitivo no pierde su condición de inteligible y útil, por ejemplo, porque el alumno atienda a una explicación, o bien observe una sola vez un fenómeno contraintuitivo. El cambio de estatus (satisfactorio, inteligible, verosimil, útil) de un esquema conceptual tleva tiempo; las implicaciones que ello tiene para la enseñanza serán tratadas más adelante.

\section{APLICACION DEL MODELO A UN CASO CONCRETO DE APRENDIZAJE DE LA FISICA}

Uno de los resultados hallados en estudios sobre aprendizaje de la Física servirá para ilustrar la aplicación del modelo PSHG. Viennot (1979), Clement (1982) y Peters (1982) entre otros, han encontrado que hay alumnos de E. Media y primeros cursos de Facultad que conservan acerca del movimiento y sus causas un esque. ma intuitivo pre-galileano (1), algunas de cuyas «leyes» son:

- para que un objeto se mueva hay que aplicarle una fuerza continua

- la fuerza aplicada coincide siempre con la dirección del movimiento 
- una fuerza constante produce una velocidad constante.

Que se pueden resumir en las expresiones $(\mathrm{v}=\mathrm{cte}$. $\mathrm{si}$ $\mathrm{F}=0 ; \mathrm{F}=\mathrm{ka}$ ).

Este esquema intuitivo es inteligible y útil porque constituye lo que Schutz y Luckmann (1973) denominarian una asociación natural entre conceptos. En primer lugar fuerza y velocidad son conceptos naturales, ya que se van formando en la infancia, desde que el niño comienza a percibir variaciones más o menos rápidas en la posición de los objetos y a tener experiencias directas con fuerzas, (Piaget, 1972 y 1973b). En segundo lugar, la observación de los movimientos cotidianos le lleva por inducción a establecer la relación causa-efecto entre las dos caracteristicas que percibe como más prominentes en el movimiento de un objeto, la causa que lo origina, la fuerza (con todas las interpretaciones alternativas que puede hacer sobre su naturaleza) y la rapidez del cambio de posición del móvil, la velocidad. Tercero, como en buena parte de los movimientos familiares actuan las fuerzas de rozamiento, no observables directamente, el alumno establece una relación intuitiva del tipo «si $F=c t e ., v=c t e . » ;(F=k v)$. Ley que utilizará en adelante para interpretar y predecir los movimientos que observe, ya que seguirá viviendo en un mundo con rozamiento.

Cuando accede al estudio formal de la Dinámica, de acuerdo con las leyes del movimiento de Newton, $1^{\text {a }}$ y $2^{a}$, el alumno tiene que aprender:

- un objeto puede mantenerse en movimiento rectilíneo uniforme si no actúan fuerzas exteriores (o si su resultante es nula)

- la fuerza aplicada puede coincidir o no con la dirección del movimiento

- una fuerza constante produce una aceleración constante.

Que se pueden resumir en las expresiones ( $\mathrm{v}: \mathrm{cte}$, si $\mathrm{F}=0 ; \mathrm{F}=\mathrm{k} a$ ).

Según el modelo PSHG aprender significativamente la $1^{\text {a }}$ y $2^{\text {a }}$ ley del movimiento, implica sustituir un esquema previo $(v=0$ si $F=0 ; F=k v)$ por otro nuevo $(v=$ cte. si $F=0 ; F=k a)$; ello requiere que el alumno llegue a considerar el nuevo esquema conceptual inteligible, verosímil y más útil que el anterior para interpretar los movimientos que observa.

La $l^{\text {a }}$ ley $(\mathrm{v}=$ cte. si $\mathrm{F}=$ o) puede ser inteligible, pero resulta increible para el alumno debido al rozamiento.

La $2^{\text {a }}$ ley $(F=k a)$ puede encontrar dificultad para reunir las tres condiciones exigidas. Primero, el concepto de aceleración no es tan asequible desde la experiencia directa como la noción de velocidad; se perciben con facilidad cambios de posición y velocidad (2) en un intervalo de tiempo apreciable, pero no ocurre lo mismo con los cambios instantáneos de la velocidad; de aqui que el alumno le resulte dificil entender el concepto de aceleración, no cumpliéndose el primer requisito para que la ley sea inteligible; en consecuencia será poco probable que el alumno pueda estructurar ta información contenida en el enunciado de la ley y captar su significado. Segundo, la ley también está en conflicto con la experiencia diaria del alumno, que a su vez la interpreta satisfactoriamente con su esquema intuitivo; hasta que la ley deje de ser contraintuitiva y le parezca útil, será dificil que el alumno la acepte.

De acuerdo con el modelo PSHG no se dan los requjsitos para el cambio conceptual, luego no es de extranat que se conserve el esquema intuitivo y no se sustituya por el nuevo. Puede suceder, y asi se ha encontrado en las investigaciones mencionadas, que coexistan los dos esquemas aisladamente; (Viennot, 1979) encontró alumnos que aplicaban correctamente la ley $\mathrm{F}$ $=$ ma a la resolución de problemas numéricos senci. llos pero utilizaban la ley intuitiva $F=k v$ para interpretar movimientos familiares para el alumno. Esta coexistencia prueba que no ha habido aprendizaje significativo, si aprender es llegar a entender y aceptar ideas nuevas que contradicen el esquema intuitivo del alumno.

\section{ESTRATEGIAS PARA FAVORECER EL APRENDIZAJE}

Varias son las propuestas que hacen los autores para facilitar el cambio conceptual que supone el aprendizaje:

a) Detectar la existencia de ideas intuitivas, sus características, variedad, persistencia y efectos sobre e! aprendizaje; para ello se requiere que el profesor disponga de tiempo e instrumentos de diagnóstico adecuado.

b) Proporcionar un número suficier:e de anomalias. Experimentos, demostraciones, problemas, ejemplos contraintuitivos, cuestiones sobre situaciones límite, si están cuidadosamente diseñados, pueden ser ocasión de que el alumno compruebe que sus ideas intuitivas no le sirven para interpretar lo que ocurre, creando un conflicto cognitivo entre sus ideas y estas anomalías; el resultado puede ser la insatisfacción y la falta de confianza en su esquema conceptual intuitivo, que perderá resistencia, hacien. do posible el cambio.

c) Utilizar analogias y modelos adecuados junto a diversos modos de representación (verbal, matemática, gráfica), familiarizando el alumno en el uso indistinto de ellos, para facilitar la comprension de las ideas nuevas.

d) Desarrollar técnicas de evaluación que permitan seguir el proceso de cambio conceptual. 


\section{POSIBLES RESULTADOS DEL PROCESO DE ENSENAANZA-APRENDIZAJE}

Se han enumerado las estrategias que el modelo PSHG propone para facilitar el cambio conceptual que supone el aprendizaje. Puede suceder, no obstante, que no haya cambio o bien que éste no sea el apropiado. Como resultado de la interacción de las ideas previas del alumno con el contenido formal de la asignatura (ideas nuevas), puede ocurrir:

$1^{\circ}$ Una idea nueva se rechaza porque no cumple alguno de los requisitos exigidos y el alumno mantiene su idea previa, que conserva su poder explicativo. Ast, la idea intuitiva «la corriente eléctrica que fluye de la bateria se va gastando» prevalece frente al concepto de corriente eléctrica como flujo de carga por unidad de tiempo, que puede ser poco inteligible por el carácter abstracto de su enunciado.

$2^{\circ}$ Una idea nueva se memoriza. Si un concepto nuevo no es inteligible, en vez de rechazarlo, puede memorizarse; el alumno es capaz de recitarlo e incluso aplicario a la resolución de problemas, cuyo algoritmo matemático se ha adquirido mecánicamen. te por repetición continuada, pero le es dificil transferir el concepto a una situación nueva (Para un estudio del tema véase Gil, 1983). Situación nueva, que no resuelve, o bien trata de hacerlo utilizando su esquema intuitivo. Esta coexistencia de ideas previas y nuevas, formando subesquemas conceptuales aislados, indica que no se ha producido aprendizaje significativo. Los resultados obtenidos por Viennot (1979), ya comentados, constituyen un ejemplo representativo de esta situación.

$3^{\circ}$ Una idea nueva se incorpora al esquema previo. Esto es lo que el modelo llama asimilación. Hay que considerar dos posibilidades:

- Las ideas previas del alumno no son erróneas desde el punto de vista científico; en este caso la incorporación de nuevos conocimientos es una forma válida de aprendizaje.

- Las ideas previas del alumno son falsas desde el punto de vista científico; la asimilación de una idea nueva, supone, en este caso, una interpretación equivocada de la misma para que sea coherente con sus ideas intuitivas; este proceso puede ser el ortgen de numerosos errores conceptuales, a veces inexplicables para el profesor. Así, un alumno puede interpretar dos ideas nuevas para èl

"el agua se descompone en oxígeno e hidrógeno" "el aire es una mezcla de oxígeno y otros gases» relacionándolas con su idea intuitiva «las burbujas de agua hirviendo son burbujas de aire», dando ori- gen al error conceptual «el agua se descompone al hervir en oxígeno e hidrógenon.

Cuando una idea nueva se asimila al esquema intuitivo falso, se produce un cambio conceptual, pero no es el adecuado, ya que surge un error conceptual y no hay por tanto aprendizaje significativo.

$4^{\circ}$ Una idea nueva sustituye a una idea intuitiva falsa. Este es el verdadero cambio conceptual que representa el aprendizaje significativo de una idea nueva que está en conflicto con una idea intuitiva: para ello se han de cumplir las condiciones ya descritas (insatisfacción con la idea intuitiva, y que la idea nueva sea inteligible verosimil y útil).

Probada la existencia de esquemas intuitivos que difieren de los esquemas conceptuales que los alumnos tienen que aprender, el resultado que se debe esperar de un proceso eficaz de enseñanza es el cambio conceptual descrito en el cuarto caso.

\section{INTERROGANTES Y PERSPECTIVAS}

Para finalizar un breve comentario sobre las implicaciones para el proceso de renovación de la enseñanza de las ciencias, tema de tal relieve que requiere un tratamiento aparte.

La evidencia acumulada en los últimos años sobre la importancia de las ideas intuitivas en el proceso de aprendizaje, ha abierto nuevos interrogantes y áreas de investigación. En la actualidad se cuestiona la adecuación de los contenidos de los programas y la eficacia de las técnicas docentes utilizadas, en especial el papel de trabajo experimental de laboratorio y/o campo. Constituyen áreas de investigación muy fructiferas, entre otras, la incidencia de los modos de pensamiento de los alumnos en la adquisición del conocimiento y la elaboración, contrastación y uso de los instrumentos para diagnosticar las ideas intuitivas y desvelar el origen de los errores conceptuales.

Asimismo se considera necesario dar un nuevo enfoque a la formación del futuro profesor de ciencias, familiarizandolo con el modo peculiar de entender la realidad del alumno, entrenándolo en la detección de errores y en el uso de estrategias que favorezcan el cambio conceptual, cosa que ya se realiza de forma regular en países como Inglaterra (STEP, 1974) o Israel (Nussbaum, 1981), por mencionar dos casos concretos.

(1) Esquema que tambien se ha encontrado en alumnos de COUf en la investigación en curso mencionada.

(2) Diferentes investigadores han encontrado evidencia de estructuras cerebrates que detectan directamente la velocidad. Hubel y Wiesel (1968) y Sexuler (1975).

\section{REFERENCIAS BIBLIOGRAFICAS}

AUSUBEL, D., 1968, Educational Psychology (N. York, Holt Rinehart \& Winston).
BEL TH, M., 1977, The process of thinking (N. York, Dand Mc Kay). 
BLACK, M y otros 1976, La justificación del razonamiento inductivo (Madrid, Alianza Universidad).

BRUNER, J.S., 1966, Toward a theory of instruction (Cambridge, Harward University Press.)

CARRASCOSA, I., 1983, Errores conceptuales en la ensehanza de las ciencias: selección bibliográfica, Enseñanza de las ciencias, vol. 1 pg. 63.65.

CLEMENT, J. 1978, Sudent responses to questions on the concept of energy. Mimegraph. (Dep. of Physics and Astronomy, University of Massachusetts).

CLEMENT, J., 1982, Students' preconceptions in introductory mechanics, Am. J. Phys. 50 (1) pp. 66-71.

DRIVER, R., 1981, Pupils' alternative frameworks in science, Eur. J. Sci. Educ., vol. 3 pp. 93-101).

EASLEY, J. \& DRIVER, R., 1978, Pupils and paradigms a review of the literature related to concept development in adolescent science students, Stud. Sci. Educ., 5 pp. $6 !-84$

EINSTEIN, A. \& INFELD, L., 1963, La Física aventura del pensamiento (Buenos Aires, Losada).

GIL, D., 1983, Tres paradigmas básicos en la ensenanza de las ciencias, Enseñanza de las ciencias, vol. 1 pg. 26-33.

GILBERT, J.K., 1982, Children's science and its consequences for teaching, Sci. Educ. 66(4) pp. 623-633.

GILBERT, J.K., 1983, Alternative conceptions. Paper presented at the AAPT Winter Meeting, N. York.

HANSON, N.R. 1977, Patrones de descubrimiento. Obser. vación y explicación, (Madrid, Alianza Universidad).

HEWSON, P.W., 1981, A conceptual change approach to learning science, Eur. J. Sci. Educ., vol. 3 pp 383-396.

HUBEL, D.H. \& WIESEL, T.N. 1968, Receptive fields and functional architecture of monkey striate conex, Journal of Physiology, 195 pp. 215-243.

KELLY, G.A., 1971, Clinical Psychology and Personality, in B. Maher (Ed.), The selected papers of George Kelly (London, John Wiley).

KUHN, T.S., 1971, La estructura de las revoluciones cientificas, (Madrid, Fondo de Cultura Económica).

LINKE, R.D. y VENZ, M.I. 1979, Misconceptions in Physical science among non-science background students, $R e$ search in Science Education, vol. 9, pp. 103-109).

MULKAY, M.J., 1972, The social process of innovation, (London, The Mc. Millan Press).

NUSSBAUM, J. 1981. Towards the diagnosis by science teachers of pupils' misconceptions: an exercise with student teachers, Eur. J. Sci. Educ., vol. 3, pp. 159-169.
OSBORNE, R.J., BELL, B.E. \& GILBERT, J.K., 1983, Science teaching and children's views of the world, Eur. J. Sci. Educ., vol. 5, pp. 1-14.

OSBORNE, R.J. \& WITTROCK, M.C., 1983, Learning science: A generative process, Sci. Educ. 67(4), pp. 479-508.

PETERS, P.C., 1982, Even honours students have conceptual difficulties with Physics, Am. J. Phys., 50(6), pp $501-508$.

PIAGET, J., 1965, La construcción de lo real en el niño, (Buenos Aires, Protes).

PIAGET, J., 1972, Les notions de mouvement ei de vitesse chez l'enfant, (Paris, PUF).

PIAGET, J.. 1973a, Las explicaciones causales (Barcelona, Seix y Barral).

PLAGET, J., 1973b, La formation de la notion de force, (Paris, PUF).

POSNER, G.J., STRIKE, K.A., HEWSON, P.W. \& GERTZOG, W.A., 1982, Accommodation of a scientific conception: Toward a heory of conceptual change, Sci. Educ. $66(2)$, pp. $211-227$.

RUSSEL, B., 1965, On the philosophy of science, (N. York, Bobbs-Merrill, Co).

SCHEFFLER, L., 1965, Conditions of knowledge, (Illinois, Scott \& Foreman, $\mathrm{Co}$ ).

SCHUTZ, A.\& LUCKMANN, T., 1973, The structures of the life world, (London, Heinemann).

SEKULER, R., 1975, Visual motion perception, in E.C. Carterette (Ed): Handbook of perception, vol. V. (N. York, Academic Press).

SMITH, E.E. 1978, Theories of semantic memory, in W. Estes (Ed.): Hondbook of learning and cognitive processes, vol. 6 (N. York, Hillsdale LEA).

STAVY, R. \& BERKOWITZ, B., 1980, Cognitive conflict as a basis for teaching quantitative aspects of the concept of temperature, Sci. Educ., 64(5), pp. 679-692.

STEP, 1974, The Science teacher education project, (Lon don, The British Council).

TASKER, C.R. 1981, Children's view and classroom expe riences, Aust. Sci. Teach. J., 27(3), pp. 51-57.

TOULMIN, S., 1972, Human Understanding, (Princeton, Princeton University Press).

VIENNOT, L., 1979, Spontaneous reasoning in elementary dynamics, Eur. J. Sci. Educ., vol. 1 pp. 205-22I.

WATTS, D.M., 1983, A study of school childrer's alternative frameworks of the concept of force, Eur. J. Sci, Educ., vol. 2, pp. 217-230. 